\title{
Compact focusing grating couplers for silicon horizontal slot waveguides
}

\author{
R. A. Larrea, ${ }^{1}$ A. M. Gutiérrez, ${ }^{1}$ J. Hurtado, ${ }^{1}$ J. M. Ramírez, ${ }^{2,3}$ B. Garrido, ${ }^{2}$ and P. Sanchis ${ }^{1, *}$ \\ ${ }^{1}$ Nanophotonics Technology Center, Universitat Politècnica València, Camino de Vera s/n Ed 8F, 46022 Valencia, Spain \\ ${ }^{2}$ MIND-IN2UB, Departament d'Electrònica, Universitat de Barcelona, Martí i Franquès 1, E-08028 Barcelona, Spain \\ ${ }^{3}$ Currently at Centre de Nanosciences et de Nanotechnologies, Université Paris Sud, CNRS, Université Paris Saclay, 91405 Orsay, France \\ ${ }^{*}$ Corresponding author: pabsanki@ntc.upv.es
}

Received 17 November 2016; revised 22 December 2016; accepted 30 December 2016; posted 3 January 2017 (Doc. ID 280802);

published 24 January 2017

In this Letter, we present the design, simulation (2D and 3D), fabrication, and experimental characterization of compact and fully etched focusing gratings for a horizontal slot waveguide based on a silicon nitride layer sandwiched between amorphous silicon and a silicon-on-insulator. The measured coupling losses are about $4 \mathrm{~dB}$ with a $3 \mathrm{~dB}$ bandwidth of $38 \mathrm{~nm}$. The fully etched configuration allows the fabrication in a single lithography step. (C) 2017 Optical Society of America

OCIS codes: (050.2770) Gratings; (130.3120) Integrated optics devices; (060.2420) Fibers, polarization-maintaining; (230.7370) Waveguides; (250.5300) Photonic integrated circuits.

https://doi.org/10.1364/OL.42.000490

During the last decade standard silicon-on-insulator (SOI) technology has emerged as the best platform for CMOS integration optics. However, with the growing attention and great interest in silicon photonics on-chip and optical interconnects, the target of high-density integration is a continuous must. In this way, the vertical integration of multiple layers of photonic components can overcome the limited bandwidth density of a single-layer photonic integrated circuit (PIC) [1]. Multi-layer silicon PICs with material films deposition or direct wafer/ sample bonding yielding horizontal slot waveguides are promising solutions. Horizontal slot waveguides, where light confinement happens in a thin low refractive index layer sandwiched by thick high refractive index materials, allow for ultra-high optical confinement, light guiding, lateral extraction of light for on-chip distribution, light emitting devices, efficient nonlinear performance and, as mentioned above, multilayer functionalities allowing the combination of several optical layers or even of electronic and optical layers [2-6]. Among the materials involved in slot waveguides, amorphous silicon as a deposited material is a particularly promising approach for enabling the desired matching between on-chip optical layers, or even the integration with electronic ones, as it allows amorphous silicon to be deposited at a low temperature [by a well-suited CMOS-compatible plasma enhanced chemical vapor deposition (PECVD) process] which is an essential requirement in CMOS fabrication processes $[7,8]$. Such multilayer technology and, consequently, high-density integration requires a high-dimensional reduction of on-chip components and, therefore, the coupling between them and between these components and optical fibers has become a nontrivial task. Therefore, for large-scale integration, light couplers with low losses, large bandwidths, small footprints, and integration compatibility are highly desirable. From all the methods proposed for efficient light coupling, grating couplers are one of the most promising solutions, since optical coupling is achieved by vertical alignment not requiring polished facets. Grating couplers can be fabricated in a CMOS-compatible process and can be placed anywhere across the wafer, enabling wafer scale testing of the integrated circuits [9]. In addition, they are very compact and have a large optical bandwidth [10-13].

Many works on grating couplers based on conventional silicon waveguides have been reported showing high efficient coupling with fully etched slots and optimized shapes as nonuniform or sub-wavelength patterns [14-18]. In horizontal slot waveguides, linear gratings have been theoretically analyzed [19] and experimentally demonstrated with coupling efficiencies of $20 \%$ [20] and, recently, 60\% [21]. However, conventional linear gratings require a few hundred micron long adiabatic taper to convert the optical mode from the grating to the waveguide with low loss resulting in a large footprint of the total structure (input grating, waveguide, and output grating). In order to reduce such length and, therefore, the device footprint yielding a higher degree of integration, the gratings can be designed in a curved shape to focus the optical mode from the grating directly into the waveguide, avoiding the adiabatic taper $[22,23]$. Focusing grating couplers to efficiently couple light into integrated circuitry have been reported with a coupling loss of $4 \mathrm{~dB}$ in a partially buried rib multilayer waveguide [24]. However, to the best of our knowledge, no focusing grating couplers for fully etched horizontal slot waveguides have been yet reported. In this Letter, a fully etched slot focusing grating is demonstrated, thus allowing a CMOS-compatible fabrication in a single lithography step (not allowed in conventional shallow-etched gratings, in which an additional lithography step is required) and compact device footprints.

The phase-matching condition that governs the first-order diffraction of a conventional linear grating coupler is expressed as

$$
k_{0} n_{\mathrm{eff}}-k_{0} n_{c} \sin (\theta)=\frac{2 \pi}{\Lambda},
$$


where $k_{0}=2 \pi / \lambda_{0}$ is the wavenumber in vacuum and $n_{\mathrm{eff}}$ is the grating effective index [related with the filling factor $(f f)$ and effective indices of etched $\left(n_{e}\right)$ and unetched regions $\left(n_{u}\right)$ as $n_{\mathrm{eff}}=(1-f f) \cdot n_{e}+f f \cdot n_{u}, n_{c}$ is the upper-cladding material refractive index, $\theta$ is the incident angle between the fiber and the grating surface normal (which in our case is fixed to $10^{\circ}$ ), and $\Lambda$ is the grating period. Then, according to [22], such a phasematching condition [Eq. (1)] can be expressed as follows to produce a curved cylindrically wavefront and focus the light in the center of curvature:

$$
\sqrt{x^{2}+z^{2}} k_{0} n_{\mathrm{eff}}-z k_{0} n_{c} \sin (\theta)=2 \pi q,
$$

where the top surface of the waveguide is chosen to lay in the $(x, z)$ plane of a right-handed Cartesian coordinate system, with $z$ along the waveguide axis and the origin chosen to be in the desired focal point; $q$ is an integer indicating the order of a curved grating line. From Eq. (2), we can derive

$$
\frac{\left(z-\frac{q \lambda_{0} n_{c} \sin (\theta)}{n_{\mathrm{eff}}^{2}-n_{c}^{2} \sin ^{2}(\theta)}\right)^{2}}{\left(\frac{q \lambda_{0} n_{\mathrm{eff}}}{n_{\mathrm{eff}}^{2}-n_{c}^{2} \sin ^{2}(\theta)}\right)^{2}}+\frac{x^{2}}{\left(\frac{q \lambda_{0}}{\sqrt{n_{\mathrm{eff}}^{2}-n_{c}^{2} \sin ^{2}(\theta)}}\right)^{2}}=1,
$$

which indicates that curved grating lines are a set of confocal ellipses with the common focal point at the origin.

The proposed horizontal slot waveguide grating structure is depicted in Fig. 1. The slot is constructed over a standard commercial SOI wafer with $220 \mathrm{~nm}$ of silicon and $2 \mu \mathrm{m}$ of buriedoxide BOX. The cross section of the waveguide has a silicon nitride layer $\left(\mathrm{Si}_{3} \mathrm{~N}_{4}\right)$ corresponding to the slot region sandwiched by two silicon slabs (the crystalline silicon layer of the SOI wafer and the deposited amorphous silicon, a-Si, layer). The refractive indices used for simulations are $n_{\mathrm{Si}_{3} \mathrm{~N}_{4}}=2$, $n_{\mathrm{Si}}=n_{\mathrm{a}-\mathrm{Si}}=3.48$ and $n_{\mathrm{SiO}_{2}}=1.46$. As a fully etched configuration is presented, the grating etching depth is the sum of the amorphous silicon, silicon nitride, and silicon layer thicknesses. Moreover, the widths of both the grating and the slot waveguide were fixed to $12 \mu \mathrm{m}$ and $300 \mathrm{~nm}$ (to ensure a singlemode condition), respectively. The polarization of light is TM, while the considered wavelength is $1550 \mathrm{~nm}$.

Both 2D and 3D finite-difference time-domain (FDTD) simulations from a commercial software [25] were used to design the grating couplers. We applied the $2 \mathrm{D}$-optimized design parameters obtained for linear gratings to the 3D simulations of the curved ones, translating the obtained period and filling factor to $n_{\text {eff }}$ by using the Bragg condition. For the 2D optimization of linear gratings, two design parameters of the grating structure are optimized: the grating period, $\Lambda$, and the filling factor, $f f$ (Fig. 2). Furthermore, three other parameters are involved in the multilayer structure of the slot waveguide: the thicknesses of the $\mathrm{Si}_{3} \mathrm{~N}_{4}$ slot, $t_{\text {slot }}$, the upper a-Si layer, $t_{\mathrm{a}-\mathrm{Si}}$ (Fig. 1), and the $\mathrm{SiO}_{2}$ uppercladding, $t_{\text {cover }}$ (Fig. 2) have been taken into account and optimized to maximize the coupling efficiency. An output grating

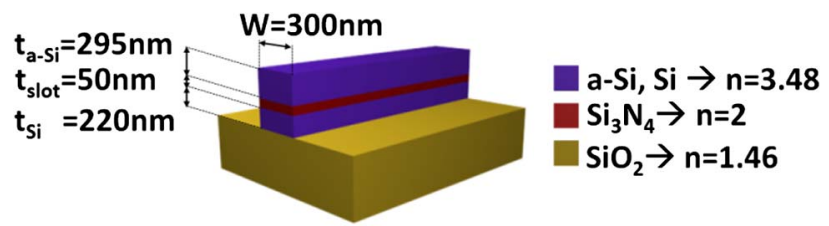

Fig. 1. Cross section of the proposed horizontal slot waveguide. The materials, layer thicknesses, and refractive indices are displayed. coupler configuration has been optimized by 2D simulations. Therefore, the TM fundamental mode was launched within the $300 \mathrm{~nm}$ width slot waveguide and then diffracted outside by the grating. The overlap integral was calculated on top of the grating to obtain the power coupled to the fundamental mode of a standard single-mode fiber (SMF) (with a MFD $=10.4 \mu \mathrm{m}$ ).

For the design, two cases were analyzed: when the two silicon slabs are symmetric in terms of thickness and when they are asymmetric. In both cases, the variation of the silicon nitride layer thickness was also analyzed, and the optimization of $f f$ and $\Lambda$ was performed. First, an optimization of the alignment parameters for the position of the output fiber $\left(S\right.$ and $\left.L_{c}\right)$ was carried out. An optimum position was obtained for $S=1 \mu \mathrm{m}$ and $L_{c}=3.5 \mu \mathrm{m}$, and these values were maintained in the whole study. Then the $t_{\text {cover }}$ effect was analyzed. It was found that coupling efficiency presents a sinusoidal shape as a function of $t_{\text {cover }}$, as expected from the grating couplers theory. One of the obtained maximums of coupling efficiency was found for $t_{\text {cover }}=1 \mu \mathrm{m}$, and such a value was set for the next simulations. Besides, in each simulation step, $\Lambda$ and $n_{\text {eff }}$ were recalculated from the Bragg diffraction Eq. (1).

The results in Fig. 3(a) show the thickness variation of the two silicon slabs and the silicon nitride layer. The highest efficiency was obtained for $t_{\mathrm{Si}}=t_{\mathrm{a}-\mathrm{Si}}=235 \mathrm{~nm}$ and $t_{\text {slot }}=50 \mathrm{~nm}$. Then, using these values for the thickness of each layer, the $f f$ and $\Lambda$ were simulated, and the results are shown in Fig. 4(a). The optimized values for $f f$ and $\Lambda$ were found to be $52 \%$ and $895 \mathrm{~nm}$, respectively. Moreover, these results show that the grating is more sensitive to period variations than to fill factor variations. Thus, the period has been optimized again, taking into account the thickness of two silicon slabs and keeping the optimal values obtained for the silicon nitride thickness layer and $f f$. The results in Fig. 5(a) show the optimal value for $\Lambda=880 \mathrm{~nm}$ and $t_{\mathrm{Si}}=t_{\mathrm{a}-\mathrm{Si}}=245 \mathrm{~nm}$, getting the highest efficiency for a symmetric case of about $44 \%$ which corresponds to a coupling loss of only $3.6 \mathrm{~dB}$.

Once the symmetric case has been analyzed, the asymmetric case was inspected to find the optimum period and fill factor, taking into account $t_{\text {cover }}, t_{\mathrm{a}-\mathrm{Si}}$ and $t_{\text {slot }}$ over $t_{\mathrm{Si}}=220 \mathrm{~nm}$ (the thickness of the standard commercial available SOI wafers). In the same way as the symmetric case, initially the coupling efficiency was obtained as a function of $t_{\mathrm{a}-\mathrm{Si}}$ and $t_{\text {slot }}$. As can be observed from Fig. 3(b), the best efficiencies are obtained for an amorphous silicon thickness value of $285 \mathrm{~nm}$ and slot thickness values between 50 and $70 \mathrm{~nm}$. A $t_{\text {slot }}=50 \mathrm{~nm}$ was chosen to maintain the thin film performance and the ultra-high optical

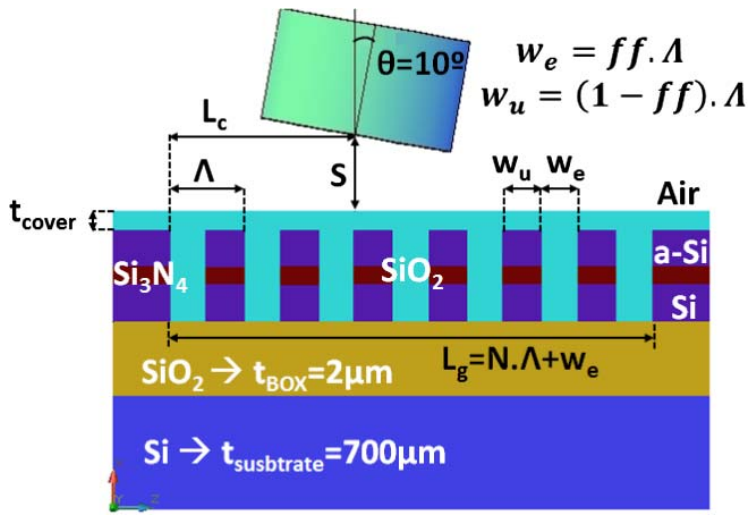

Fig. 2. Schematic of the proposed grating coupler and the main design parameters optimized by simulation. 
confinement inherent to the slot effect. Then, a coupling efficiency optimization as a function of $f f$ and $\Lambda$ was carried out. As it is shown in Fig. 4(b), the highest efficiency was obtained for $f f=56 \%$ and $\Lambda=895 \mathrm{~nm}$. Finally, again the coupling efficiency was obtained as a function of period and amorphous silicon thickness and results are plotted in Fig. 5(b). As can be observed, the highest coupling efficiency was obtained for

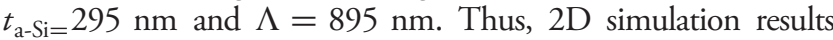
for the asymmetric case showed a coupling loss of only $2.8 \mathrm{~dB}$ $(51.72 \%)$, better than the symmetric case, for a grating with 50 and $295 \mathrm{~nm}$ and $1 \mu \mathrm{m}$ of $\mathrm{Si}_{3} \mathrm{~N}_{4}, \mathrm{a}-\mathrm{Si}$, and upper-cladding $\mathrm{SiO}_{2}$ layer thicknesses, respectively, and a period of $895 \mathrm{~nm}$ with a filling factor of $56 \%$.

Finally, a 3D simulation of the whole structure for the asymmetric case with the optimized parameters obtained through 2D simulations was performed. The obtained normalized transmission in this case is $-7 \mathrm{~dB}$ fiber to fiber, which corresponds to a coupling efficiency of about $-3.5 \mathrm{~dB}(\approx 44 \%)$ per
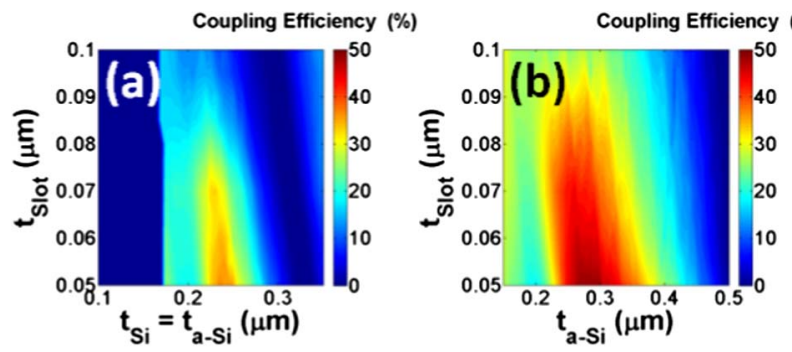

Fig. 3. $2 \mathrm{D}$ contour plot of the coupling efficiency output grating as a function of the a-Si layer and the slot layer thicknesses for the (a) symmetric and (b) asymmetric waveguides. In the symmetric case, the $\mathrm{Si}$ thickness $\left(t_{\mathrm{Si}}\right)$ is the same as in the a-Si layer while, in the asymmetric case, the Si thickness is fixed to 220 .
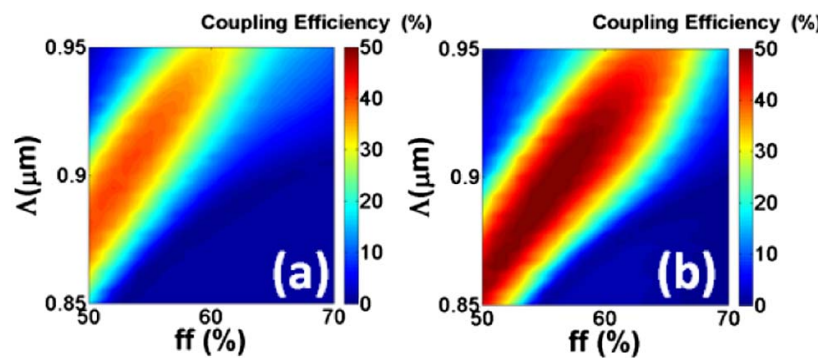

Fig. 4. $2 \mathrm{D}$ contour plot of the coupling efficiency output grating as a function of the period and the fill factor for the (a) symmetric and (b) asymmetric cases.
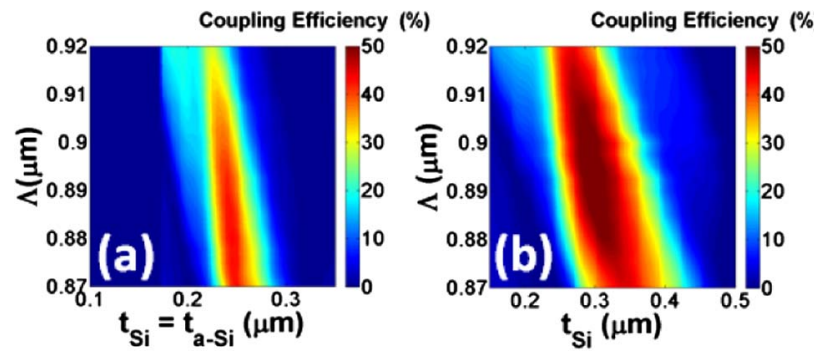

Fig. 5. $2 \mathrm{D}$ contour plot of the coupling efficiency output grating as a function of the period and Si thicknesses for the (a) symmetric and (b) asymmetric cases. grating coupler. The difference in coupling efficiency results between the $3 \mathrm{D}$ and $2 \mathrm{D}$ simulations is about $7 \%$, and it is due to the difference in the simulation resolution since $2 \mathrm{D}$ simulations were made with a resolution of $30 \mathrm{~nm}$, while the 3D simulation was made with $50 \mathrm{~nm}$.

The $\mathrm{Si}_{3} \mathrm{~N}_{4}$ thin layer, with an optimized thickness of $50 \mathrm{~nm}$ and a refractive index of 1.96 (measured at $\lambda=1550 \mathrm{~nm}$ by ellipsometry), was grown by PECVD over the crystalline silicon layer of a SOI wafer. Then, a layer of a-Si with an optimized thickness of $295 \mathrm{~nm}$ and a refractive index of 3.41 (again measured at $\lambda=$ $1550 \mathrm{~nm}$ by ellipsometry) was deposited by the same technique onto the $\mathrm{Si}_{3} \mathrm{~N}_{4}$ layer. The measured values of refractive indices are in good agreement with the values used in the simulations. The fabrication process is based on an electron beam direct writing process performed on a coated $180 \mathrm{~nm}$ hydrogen silsesquioxane (HSQ) resist film (XR-1541.06 from Dow Corning). The mentioned electron beam exposure, performed with a Vistec EBPG5000 tool, was optimized in order to reach the required dimensions employing an acceleration voltage of $100 \mathrm{keV}$ and an aperture size of $200 \mu \mathrm{m}$. After developing the HSQ resist using tetramethylammonium hydroxide, the resist patterns were transferred into the a-Si samples employing an also optimized inductively coupled plasma-reactive ion etching process with fluoride gases. Finally, a $\mathrm{SiO}_{2} 1 \mu \mathrm{m}$ upper-cladding was deposited on the samples by using a PECVD system from Applied Materials.

Figure 6 shows scanning electron microscope (SEM) images of fabricated devices. A pair of input and output focusing gratings with $36 \mu \mathrm{m}$ of focal distance was connected by a $300 \mathrm{~nm}$ width waveguide. The final fabricated focusing grating footprint was as low as $22 \times 65 \mu \mathrm{m}$, allowed by the curved shape. Moreover, waveguides with different lengths were fabricated to extract the propagation losses of the slot waveguide.

In general, fully etched gratings usually suffer from strong back Fresnel reflections into the waveguide caused by the refractive index contrast in the grating region which increases the fiber chip coupling loss. Such reflections can be mitigated by antireflective facets which in our gratings have been achieved by triangular shapes with a height of $5 \mu \mathrm{m}$ and a base of $2 \mu \mathrm{m}$, as can observed in Fig. 6, left.

To measure the coupling efficiency through the transmission spectra characterization, light is launched from a tunable broadband laser (SANTEC TSL-210F) to an external fiber polarization controller to adjust the TM polarization of the input light. The SMF fiber is positioned above the focusing input grating at $10^{\circ}$ with respect to the vertical axis to couple the light into the chip. After the waveguide, the output grating diffracts the light to another SMF fiber (also at $10^{\circ}$ ), which is connected to an optical
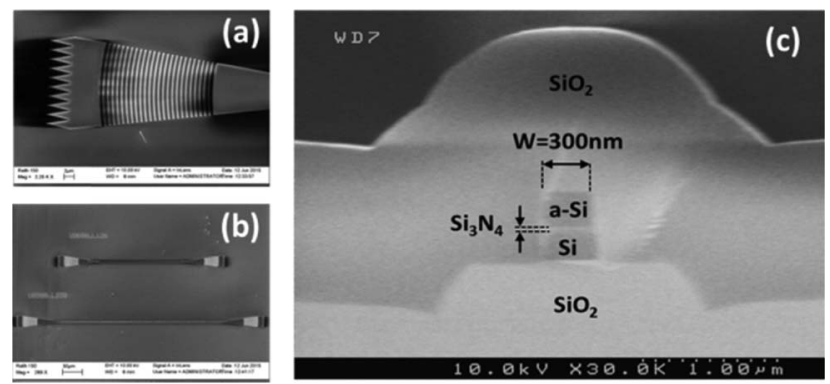

Fig. 6. SEM images of the (a) fabricated focusing grating couplers and (b) slot waveguides with different lengths and (c) slot waveguide cross section. 
Table 1. Parameter Deviations Suffered During the Fabrication Process

\begin{tabular}{lcc}
\hline & Designed & Measured \\
\hline$\Lambda$ & $895 \mathrm{~nm}$ & $902 \mathrm{~nm}$ \\
$f f$ & $56 \%$ & $69 \%$ \\
$t_{\text {slot }}$ & $50 \mathrm{~nm}$ & $40 \mathrm{~nm}$ \\
$t_{\text {a-si }}$ & $295 \mathrm{~nm}$ & $300 \mathrm{~nm}$ \\
\hline
\end{tabular}

power meter (Thorlabs, PM320E). It should be noticed that the measured grating spectrum is shifted with respect to the operation wavelength $(1550 \mathrm{~nm})$, as it is centered at about $1480 \mathrm{~nm}$. This wavelength shift could be originated due to the deviations of the grating design parameters (which affect the Bragg condition), as well as changes in the multilayer structure (which affect the effective index and, therefore, also the Bragg condition), suffered during the fabrication process. The higher deviation in the filling factor is mainly responsible for the wavelength shift of the transmission spectrum. As feedback, new simulations were carried out, taking into account such deviations confirming the wavelength shift from about 1550 to $1480 \mathrm{~nm}$. Table 1 summarizes the above mentioned deviations.

The measured transmission spectrum of a single focusing grating (normalizing the setup and propagation waveguide losses) is shown in Fig. 7 and is compared with the simulated result. The measured coupling losses are $4.4 \mathrm{~dB}$ which are in good agreement with the simulated value. On the other hand, the measured $3 \mathrm{~dB}$ bandwidth is $38 \mathrm{~nm}$. Furthermore, a low extinction ratio less than $1 \mathrm{~dB}$ of the oscillation ripples due to back reflections has been achieved.

It is worth noting that one of the major drawbacks associated with fully etched grating couplers is the insertion losses mainly caused by the penetration loss to the substrate and the mode mismatch between the fiber and the grating. Thus, a possible approach to further enhance coupling efficiency can come from the optimization of a nonuniform grating pattern by apodizing the grating period to achieve a better mode overlap $[9,23]$ or the engineering of the buried-oxide thickness $[10,15]$, since it has a significant impact on the grating coupler performance due to the secondary reflections at dielectric interfaces.

Compact focusing grating couplers in horizontal slot waveguides have been $2 \mathrm{D}$ and $3 \mathrm{D}$ simulated and experimentally demonstrated. A horizontal slot structure with a Si3N4 thin film sandwiched between crystalline silicon and deposited

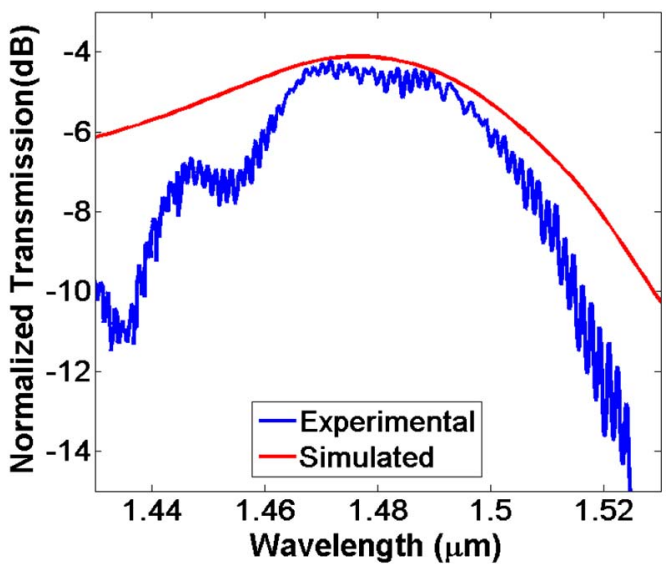

Fig. 7. Measured normalized transmission spectrum of a single focusing grating and a comparison with the simulated result. amorphous silicon layers has been fabricated with the developed fully etched gratings. Grating couplers with coupling losses of 4.4 and $3 \mathrm{~dB}$ bandwidths of $38 \mathrm{~nm}$ have been demonstrated. Reported gratings are compact due to their curved shapes, allowing low complexity in the CMOS-compatible fabrication process with a single lithography step and encouraging the multilayer integration on silicon platform.

Funding. Ministerio de Economía y Competitividad (MINECO) (TEC2012-38540-C02-02, TEC2016-76849C2-2-R); Generalitat Valenciana (NANOMET PLUS -PROMETEOII/2014/034).

\section{REFERENCES}

1. S. Zhu and G.-Q. Lo, J. Lightwave Technol. 34, 386 (2016).

2. V. R. Almeida, Q. Xu, C. A. Barrios, and M. Lipson, Opt. Lett. 29, 1209 (2004).

3. R. Sun, P. Dong, N. Feng, C. Hong, J. Michel, M. Lipson, and L. Kimerling, Opt. Express 15, 17967 (2007).

4. A. Martinez, J. Blasco, P. Sanchis, J. V. Galan, J. Garca-Ruperez, E. Jordana, P. Gautier, Y. Lebour, S. Hernandez, R. Spano, R. Guider, N. Daldosso, B. Garrido, J. M. Fedeli, L. Pavesi, and J. Marti, Nano Lett. 10, 1506 (2010).

5. A. Tengattini, D. Gandolfi, N. Prtljaga, A. Anopchenko, J. M. Ramírez, F. Ferrarese Lupi, Y. Berencén, D. Navarro-Urrios, P. Rivallin, K. Surana, B. Garrido, J.-M. Fedeli, and L. Pavesi, J. Lightwave Technol. 31, 391 (2013).

6. J. M. Ramírez, F. Ferrarese Lupi, Y. Berencén, A. Anopchenko, J. P. Colonna, O. Jambois, J. M. Fedeli, L. Pavesi, N. Prtljaga, P. Rivallin, A. Tengattini, D. Navarro-Urrios, and B. Garrido, Nanotechnology 24 , 115202 (2013)

7. S. K. Selvaraja, E. Sleeckx, M. Schaekers, W. Bogaerts, D. V. Thourhout, P. Dumon, and R. Baets, Opt. Commun. 282, 1767 (2009).

8. F. G. Della Corte and S. Rao, Trans. Electron. Devices 60, 1495 (2013).

9. L. He, Y. Liu, C. Galland, A. E.-J. Lim, G.-Q. Lo, T. Baehr-Jones, and M. Hochberg, IEEE Photon. Technol. Lett. 25, 1358 (2013).

10. D. Taillaert, W. Bogaerts, P. Bienstman, T. F. Krauss, P. Van Daele, I. Moerman, S. Verstuyft, K. De Mesel, and R. Baets, Quantum Electron. 38, 949 (2002).

11. D. Taillaert, P. Bienstman, and R. Baets, Opt. Lett. 29, 2749 (2004).

12. D. Taillaert, F. V. Laere, M. Ayre, W. Bogaerts, D. V. Thourhout, P. Bienstman, and R. Baets, J. Appl. Phys. 45, 6071 (2006).

13. L. Vivien, D. Pascal, S. Lardenois, D. Marris-Morini, E. Cassan, F. Grillot, S. Laval, J.-M. Fédéli, and L. El Melhaoui, J. Lightwave Technol. 24, 3810 (2006).

14. R. Halir, P. Cheben, S. Janz, D.-X. Xu, I. Molina-Fernández, and J. G. Wangüemert-Pérez, Opt. Lett. 34, 1408 (2009).

15. B. Schmid, A. Petrov, and M. Eich, Opt. Express 17, 18371 (2009).

16. Z. Xiao, F. Luan, T.-Y. Liow, J. Zhang, and P. Shum, Opt. Lett. 37, 530 (2012).

17. D. Benedikovic, P. Cheben, J. H. Schmid, D.-X. Xu, J. Lapointe, S. Wang, R. Halir, A. Ortega-Moñux, S. Janz, and M. Dado, Laser Photon. Rev. 8, L93 (2014).

18. R. Halir, P. Bock, P. Cheben, A. Ortega-Moñux, C. Alonso-Ramos, J. H. Schmid, J. Lapointe, D.-X. Xu, J. G. Wangüemert-Pérez, I. Molina-Fernández, and S. Janz, Laser Photon. Rev. 9, 25 (2015).

19. J. V. Galan, P. Sanchis, J. Blasco, and J. Marti, IEEE Photon. Technol. Lett. 20, 985 (2008).

20. J. V. Galan, P. Sanchis, J. Blasco, A. Martinez, J. Marti, J. M. Fedeli, E. Jordana, P. Gautier, and M. Perrin, Electron. Lett. 45, 262 (2009).

21. J. Covey and R. T. Chen, Opt. Express 21, 10886 (2013).

22. F. Van Laere, T. Claes, J. Schrauwen, S. Scheerlinck, W. Bogaerts, D. Taillaert, L. O'Faolain, D. Van Thourhout, and R. Baets, IEEE Photon. Technol. Lett. 19, 1919 (2007).

23. Y. Wang, X. Wang, J. Flueckiger, H. Yun, W. Shi, R. Bojko, N. A. F. Jaeger, and L. Chrostowski, Opt. Express 22, 20652 (2014).

24. C. Xiong, W. H. P. Pernice, M. Li, and H. X. Tang, Opt. Express 18, 20690 (2010).

25. FullWAVE from Synopsys RSoft. 